\title{
Acclimatization of Thalassiosira pseudonana Photosynthetic Membranes to Environmental Temperature Changes
}

\author{
Monika Bojko ${ }^{1}$, Monika Olchawa-Pajor', Marek Chyc², Reimund Goss ${ }^{3}$, Susann Schaller-Laudel ${ }^{3}$, \\ Dariusz Latowski ${ }^{1}$ \\ ${ }^{1}$ Department of Plant Physiology and Biochemistry, Faculty of Biochemistry, Biophysics and Biotechnology \\ Jagiellonian University \\ Gronostajowa 7, 30-387 Kraków, Poland \\ m.bojko@uj.edu.pl; olchawa.pajor@gmail.com; dariuszlatowski@gmail.com \\ ${ }^{2}$ Department of Environmental Protection, State Higher Vocational School in Tarnów \\ ul. Mickiewicza 8, 33-100 Tarnów, Poland \\ mrsch@tlen.pl \\ ${ }^{3}$ Institute of Biology, University of Leipzig \\ Johannisallee 21-23, 04103 Leipzig, Germany \\ rgoss@rz.uni-leipzig.de; schall@rz.uni-leipzig.de
}

\begin{abstract}
The greenhouse effect results in warming the planet's surface and a higher ocean heat content. It causes changes of the water circulation frequency and turbulent mixing, delivering warm water, phytoplankton nutrients and $\mathrm{CO}_{2}$ from deeper layers of water to the water surface. All of these changes can have an impact on Earth's ecosystems. In the present study the effect of changing environmental temperature on a model diatom species, Thalassiosira pseudonana was tested under laboratory conditions. The purpose of the work was to analyze the temperature effect on fluidity and chemical composition of the thylakoid membranes. The photosynthetic activity and photosynthetic pigment contents of diatoms adapted to different temperatures were also determined. Our result show decreases of the growth rate and chlorophylls concentration in diatom cells cultured at lower temperature. It was also detected that increases of the polyunsaturated and decreases of saturated fatty acids, as well as changes in lipids:proteins ratio, resulted in stabilization of the thylakoid membranes fluidity and photosynthetic efficiency ( $\mathrm{Fv} / \mathrm{Fm})$ in a physiological range of the temperature. The result show that the regulation of the concentration and interaction of fatty acids, proteins and pigments is the most important factor in the adaptation strategies of diatoms to the environmental temperature changes. Additionally, the regulation of membrane fluidity was demonstrated as important mechanism of the diatom adaptation to greenhouse effects. We also postulate that the adaptive mechanisms to temperature changes are differently expressed in the polar or hydrophobic regions of photosynthetic membranes.
\end{abstract}

Keywords: membrane fluidity, fatty acids composition, diatoms, acclimatization mechanisms, photosynthesis

\section{Introduction}

During the last 100 years the increase in ocean heat content was mainly observed during two different time periods. The first was at the beginning of the 20th century, the second began in the 1970s and continues until now. They could both be caused by anthropogenic and natural factors [1]. The warming of ocean waters as well as the influence of wind conditions cause the change of the water circulation and turbulent mixing delivers warm water, phytoplankton nutrients and $\mathrm{CO}_{2}$ from deeper layers of water to the water surface. These changes strongly influence the photosynthetic activity of phytoplankton and result both in changes of the biomass production and $\mathrm{CO}_{2}$ fixation $[1,2,3]$. One of the most important marine group of phytoplankton are diatoms (Bacillariophyta). They are the dominant organisms in extremely cold, but nutrient rich, marine environment and are responsible for around $40 \%$ of marine and $20 \%$ of global net primary production which corresponds to 20 billion ton of fixed carbon per year [3, 4]. Their chloroplasts are significantly different from chloroplasts of vascular plants. The diatom chloroplasts are surrounded by an envelope which consists of four membranes where the girdle lamella runs parallel to the envelope. The thylakoid membranes are arranged in groups of three [5, 6, 7]. Certain regions of thylakoid membranes are characterized by differences in the protein, pigment-protein and lipid 
composition. The light harvesting systems of diatoms are called fucoxanthin chlorophyll binding proteins (FCP) because they bind high amounts of fucoxanthin, $(\mathrm{Fx})$ a unique xanthophyll pigment which can be also found in brown algae and most other heterokonts. FCPs serve as antenna for PSI and PSII or as specific antenna for PSI only [5, 7, 8]. In addition to Fx, two different xanthophylls can be bound to FCPs. These are diadinoxanthin (Ddx) and diatoxanthin (Dtx). They are engaged in one of the most important photoprotective mechanism known as diadinoxanthin cycle [9, 10]. Moreover, diatom chloroplasts contain chlorophyll a $(\mathrm{Chl} \mathrm{a}), \mathrm{c} 1$ and $\mathrm{c} 2(\mathrm{Chl} \mathrm{c})$ [7, 8, 11]. Thylakoid membranes of diatoms are composed from the same lipid classes as the thylakoids of vascular plants i.e. galactolipids such as monogalactosyldiacylglycerol (MGDG) and digalactosyldiacylglycerol (DGDG), and negatively charged lipids i.e. the phospholipid phosphatidylglycerol (PG) and the sulfolipid sulfoquinovosyldiacylglycerol (SQDG). However, the proportions of these lipids are different from vascular plants. The concentration of negatively charged lipids, SQDG and PG, in diatoms are two times higher than in vascular plants [7, 12]. Furthermore, the fatty acid profile of diatom lipids is also unique. The diatom membrane lipids contain longer and more unsaturated fatty acids, e.g. 20:5 and 22:6. It is known that the decrease of growth temperature during diatom cultivation enhances synthesis of polyunsaturated fatty acids (PUFA), especially eicosapentaenoic acid (EPA) [13, 14, 15, 16].

Although the ecological significance of diatoms is comparable to that of tropical forests, there exist only few data on their acclimation mechanisms to environmental temperature changes. To study the adaptation mechanisms of diatoms to climate warming, the model marine diatom, Thalassiosira pseudonana whose whole genome has been sequenced [17], was cultured at two different temperatures. The purpose of the work was the comparative analysis of the temperature effect on diatom thylakoid membrane composition and fluidity. The photosynthetic activity and photosynthetic pigment contents of diatoms adapted to different temperatures were also determined.

\section{Materials and Methods}

The Th. pseudonana were grown under standard culture conditions in f/2 medium [18], with a photoperiod of 10: $14 \mathrm{~h}$ $\mathrm{D}$ : $\mathrm{L}$ with a light intensity of $40 \mu \mathrm{Em}^{-2} \mathrm{~s}^{-1}$ at low $\left(12{ }^{\circ} \mathrm{C}\right)$ and moderate temperature $\left(20^{\circ} \mathrm{C}\right)$. Approximately $300 \mathrm{ml}$ of inoculum with an optical density $\mathrm{OD}_{600}$ of $0.3-0.4$ was used to start a $1200 \mathrm{ml}$ culture. Cultures were shaken several times a week during the light phase to keep cells in suspension and maintain an optimal exchange of gas and nutrients [19].

Cell density was measured at $600 \mathrm{~nm}[20]$ with a spectrophotometer.

Chlorophyll fluorescence was measured with a PAM-210 fluorimeter (Waltz, Germany). Before measurements samples were collected and centrifuged to obtain pellet of the cells which were dark-adapted for 15 min [19]. Software generated Fo (minimum) and Fm (maximum) fluorescence values which were used to calculate the optimum quantum yield Fv/Fm.

Protein concentration was measured by Lowry method [21].

$\mathrm{Chl}$ a and Chl c were extracted from T. pseudonana by $90 \%$ acetone, with liquid nitrogen cooling. Concentration of the chlorophylls was determined with a spectrophotometer and calculated according to Jeffrey and Humphrey [22].

Thylakoid membranes were isolated on the eight day after inoculation according to the method described by Lepetit et al. [23].

To compare lipids:proteins ratio in the different thylakoid membranes, FT-IR spectroscopy (Fourier Transform Infrared Spectrometer, Bruker Alpha-P with Opus 6.5 software) was used.

The fluidity of freshly prepared T. pseudonana thylakoid membranes was monitored by EPR (Electron Paramagnetic Resonance) spectroscopy using two spin labels 5-SASL and 16-SASL. Samples for EPR measurements were prepared by mixing $0.1 \mathrm{mM}$ of spin labels and thylakoid membranes $(1 \mathrm{mg} / \mathrm{ml}$ of $\mathrm{Chl}$ a in final concentration). EPR spectra of spin labels as a function of temperature were recorded by Miniscope EPR (Magnettech, Germany). EPR spectral parameters

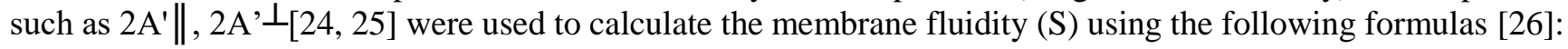

$$
\mathrm{S}=0.5407\left(\mathrm{~A}^{\prime} \|-\mathrm{A}^{\prime} \perp\right) / \mathrm{a}_{\mathrm{o}}
$$

where

$$
\mathrm{a}_{\mathrm{o}}=\left(\mathrm{A}^{\prime} \|+2 \mathrm{~A}^{\prime} \perp\right) / 3
$$


The preparation of fatty acid methyl esters (FAME) and their derivatization were carried out according to Ichihara and Fukubayashi [27]. The chromatographic analysis of n-hexane extracts was performed on Agilent 6890N gas chromatograph (Agilent Technologies, USA) equipped with a flame ionization detector (FID and capillary column IL-100 (Supelco, 28884-U). Certified reference material 37 FAME MIX (Supelco, CRM 47885) and internal standard (biphenyl) for the calibration and verification of laboratory equipment and analytical procedure were used for the determination of FAME) content.

The membrane fluidity of diatom thylakoid membranes was additionally analyzed by the laurdan (6-dodecanoyl-2dimethylaminonaphthalene) fluorescence method. For laurdan-labelling thylakoids were incubated with $1 \mu \mathrm{M}$ laurdan for $30 \mathrm{~min}$. The concentration of the thylakoids was adjusted to $2.5 \mu \mathrm{g} \mathrm{Chl} \mathrm{a} \mathrm{ml}{ }^{-1}$. Steady-state fluorescence emission spectra were recorded in the temperature range from $5^{\circ} \mathrm{C}$ to $30^{\circ} \mathrm{C}$. For the interpretation of the laurdan fluorescence signals the general polarization $(\mathrm{GP})$ value was calculated:

$$
\mathrm{GP}=(\mathrm{Ig}+\mathrm{If}) /(\mathrm{Ig}+\mathrm{If})
$$

where Ig and If are the fluorescence intensities at the emission maxima of laurdan (450 and 500nm) incorporated into a membrane in the gel and fluid phase, respectively. Laurdan excitation was set to a wavelength of $390 \mathrm{~nm}$ [28].

\section{Results and Discussion}

The survival in the changing environment depends on abilities to adapt or acclimate to stress factors such as high and low temperature. The warming climate results in untypical ocean circulation and temperature changes of water, which effect the physiological and metabolic processes of important phytoplankton species such as diatoms [3, 29]. The adaptation mechanisms of marine diatoms to different temperatures are still unclear. Also, the mechanisms protecting photosynthetic machinery of diatoms against the greenhouse effect need to be explained. For this purpose the effect of temperature on T. pseudonana growth, photosynthetic membrane properties and photosynthesis parameters was tested in batch cultures. Firstly, comparative analysis of growth kinetics of $T$. pseudonana cells, adapted to low and moderate temperature, were performed (Fig. 1). The obtained results showed statistically significant differences in the diatom growth kinetics at 12 and $20^{\circ} \mathrm{C}$. The growth at $20^{\circ} \mathrm{C}$ was faster during the first three days of culture, and the differences were slightly increasing during the next days. The growth of diatoms under both temperatures was characterized by two phases: a first linear growth phase with higher rate was observed between the first and fifth day after inoculation; the second phase with a slower growth rate was observed after the sixth day of culture. Similar results were observed for P. tricornutum cultures under the same culture conditions, where a gradual decrease of the growth rate at low temperature was determined $[19,30]$.
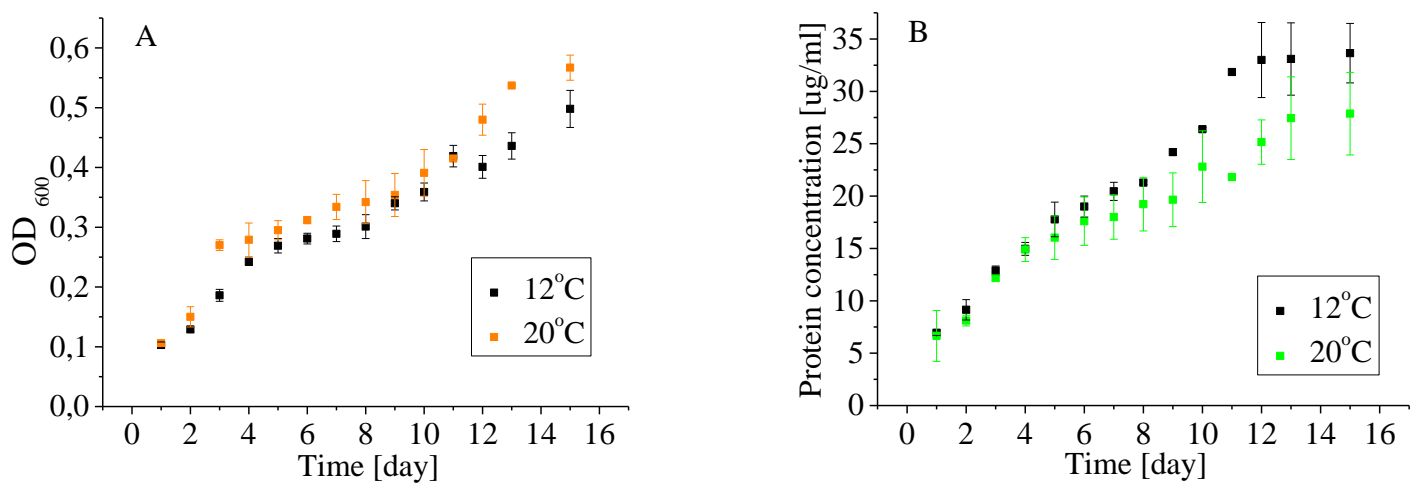

Fig. 1: Effect of temperature on OD 600 (A) and protein concentration (B) of T. pseudonanna growing at different temperature (12 and $20^{\circ} \mathrm{C}$ ) under the same light conditions $\left(40 \mu \mathrm{mol} \mathrm{m}^{-1} \mathrm{~s}^{-1}\right)$ in batch cultures. Values are the average of three experiments ( \pm standard error).

Analysis of temperature effect on $T$. pseudonanna culture growth monitored by $\mathrm{OD}_{600}$ measurements was additional supported by simultaneous determination of protein (Fig 1B), Chl a and c concentration (Fig 2) in the cells. The increase of 
the protein concentration was observed in cells cultured at both temperatures (Fig. 1B). Protein level in cells cultured at lower temperature was increasing faster than at higher temperature. After the eighth day of inoculation, the higher level of protein content was clearly visible in cells adapted to lower temperature (Fig. 1B). Additionally, the ratios of the protein level to the $\mathrm{OD}_{600}$ values or the $\mathrm{Chl}$ a concentration (Fig. 3), clearly indicate that at $12^{\circ} \mathrm{C}$ the protein concentration in diatoms was higher than at $20^{\circ} \mathrm{C}$. Observed results are in line with the data published for other cold adapted organisms. It has been postulated that at low temperatures the reduced chemical reaction rates are compensated by increasing the enzyme concentration [31].
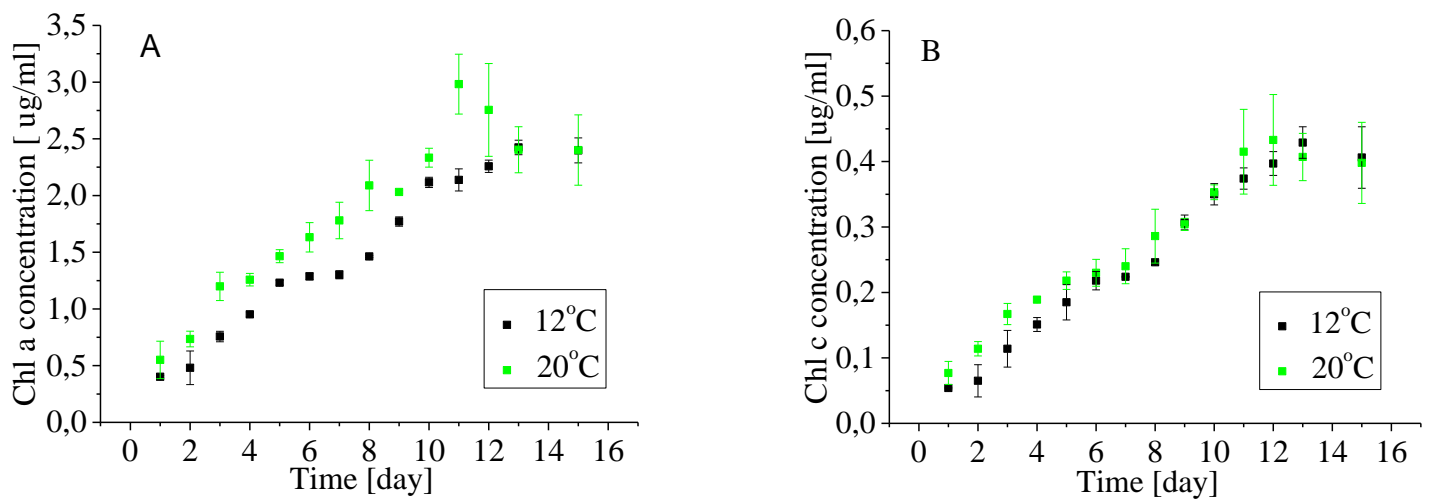

Fig. 2: Effect of temperature on chlorophyll a (Chl a) (A) and chlorophyll c (Chl c) (B) concentration in T. pseudonana at different temperatures $\left(12\right.$ and $\left.20^{\circ} \mathrm{C}\right)$ under the same light conditions $\left(40 \mu \mathrm{mol} \mathrm{m}^{-1} \mathrm{~s}^{-1}\right)$ in batch cultures. Values are the average of three experiments ( \pm standard error).

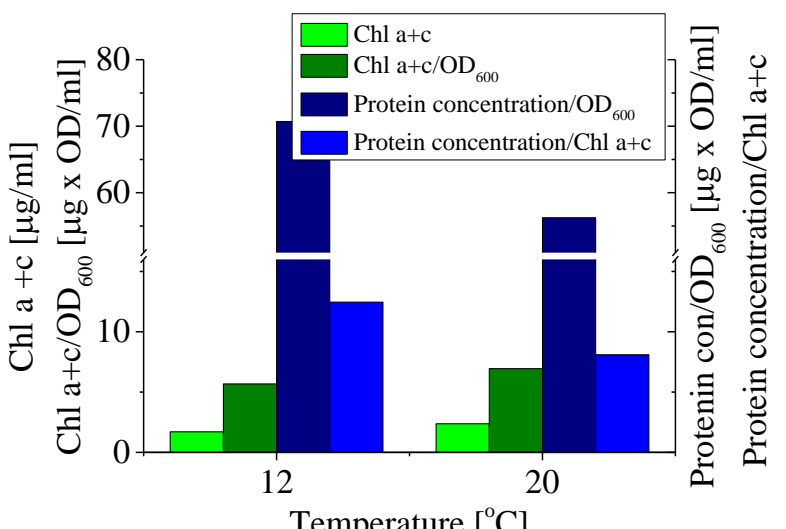

Fig. 3: Effect of temperature on T. pseudonana chlorophyll concentration and the ratio of chlorophylls to $\mathrm{OD}_{600}$ as well as on the protein to $\mathrm{OD}_{600}$ value.

Although chlorophyll levels were increasing together with the $\mathrm{OD}_{600}$ values, in contrast to the protein concentration, the chlorophyll content was slightly higher in cells cultured at $20^{\circ} \mathrm{C}$. After 12 days of cultivation, decreases of Chl a and $\mathrm{Chl} \mathrm{c}$ levels were detected at both temperatures (Fig. 2). These changes in the concentration of chlorophylls may be associated with the maintenance of the balance between the temperature independent processes of light-harvesting and the temperature-sensitive, enzymatic steps of photosynthesis. Changes in the FCP protein and pigment concentrations as the result of temperature response in diatom cells have not been reported so far.

$\mathrm{Fv} / \mathrm{Fm}$ is the most used parameter to determine stress effects on plant photosystem II (PS II) [32]. Small variability in photosynthetic efficiency of PSII was demonstrated in diatoms cultivated at low and moderate temperature (Fig. 4). Fv/Fm increased faster in cells growing at $20^{\circ} \mathrm{C}$. The level of $\mathrm{Fv} / \mathrm{Fm}$ achieved the maximum value of around 0.72 during the first 5 days of culture. The highest $\mathrm{Fv} / \mathrm{Fm}$ for diatoms growing at $12^{\circ} \mathrm{C}$ was reached on the $8^{\text {th }}$ day of culture and it was slightly lower than Fv/Fm detected for diatoms at $20^{\circ} \mathrm{C}$. Subsequently, Fv/Fm values of both culture types were decreasing. 
Additionally, the Fv/Fm values became comparable in cells cultured at both tested temperatures. The period of the gradually decreasing Fv/Fm values was not correlated with period of the decline in the Chl concentration. This phenomenon was not observed in P. tricornutum in our previous experiments [19].

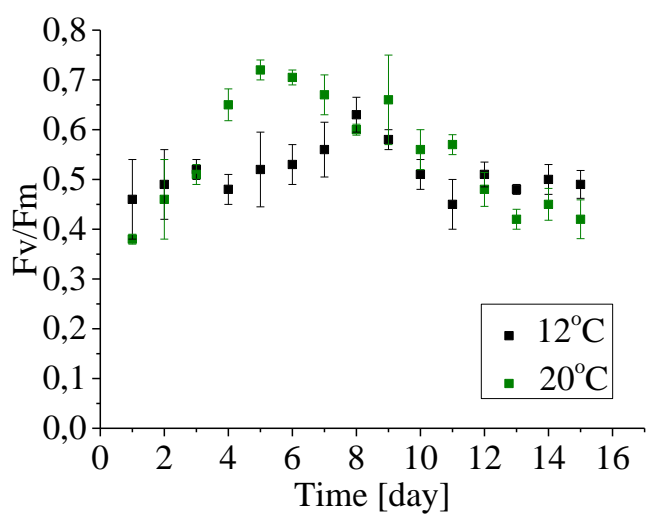

Fig. 4: Effect of temperature on Fv/Fm of T. pseudonana growing at different temperature $\left(12\right.$ and $\left.20^{\circ} \mathrm{C}\right)$ under the same light conditions $(40 \mu \mathrm{mol} \mathrm{m}-1 \mathrm{~s}-1)$ in bath cultures. Values are the average of three experiments ( \pm standard error).

In the photosynthetic machinery the D1 protein (also known as PsbA) is most prone to photooxidative damage under high light conditions. Because D1, together with the D2 protein forms the reaction core of PSII, these proteins are rapidly renewed in a turnover repair cycle [33]. However, decrease of D1 protein level in diatoms was also observed under low light $\left(40 \mu \mathrm{mol} \mathrm{m} \mathrm{m}^{-1} \mathrm{~s}^{-1}\right)$ conditions [32]. This light intensity is comparable to light conditions of T. pseudonana cultures in the present experiments. Decrease of Fv/Fm indicates a lower activity of the PS II reaction center, probably caused by insufficient D1 turnover [32]. It is known that the D1 re-synthesis is regulated by unsaturated fatty acids and thylakoid membrane fluidity. The moderate or low temperature inhibits the repair of PSII but does not have an effect on photodamage $[34,35]$. T. pseudonanna adaptation to different temperatures probably results in comparable rates of D1 protein re-synthesis at both temperatures tested in the present experiments.
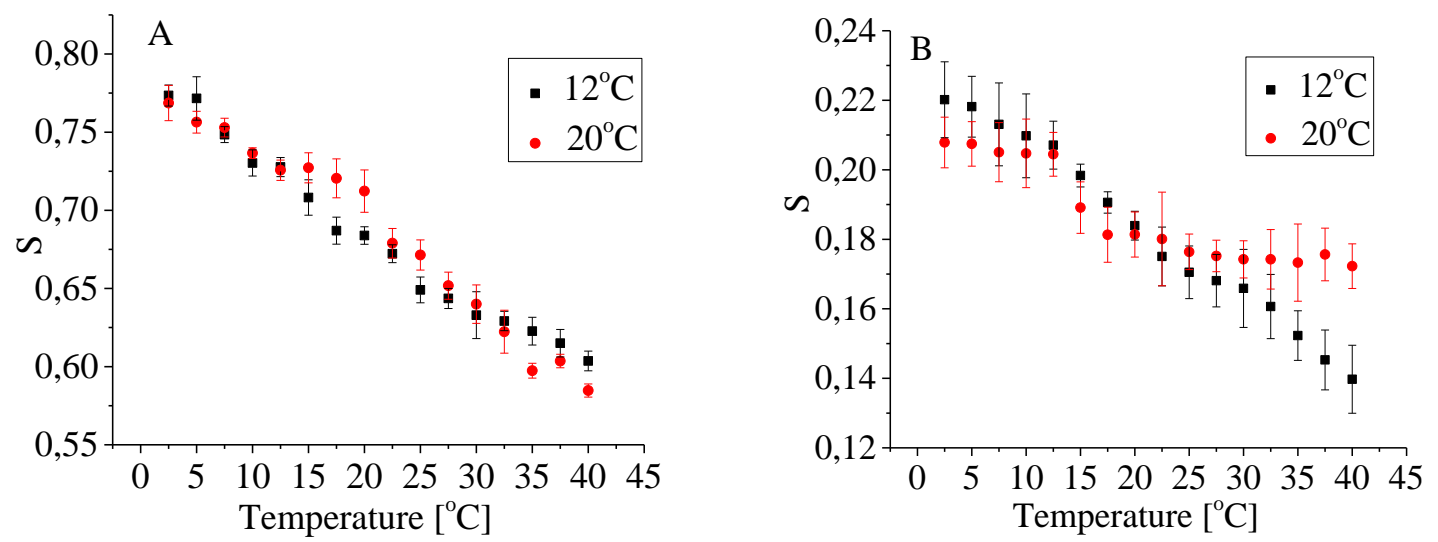

Fig. 5: Growth temperature effect on temperature dependence of $\mathrm{S}$ (fluidity) parameter calculated for T. pseudonana thylakoid membranes labelled with 5-SASL (A) and 16-SASL (B). Results for the one representative isolation. Confidence intervals for the level of significance $\alpha=0,05$.

To analyze the acclimatization mechanism of diatom photosynthetic membranes to changing temperatures, we isolated the thylakoid membranes on the eighth day after inoculation when diatom cultures were in the growth phase and the Fv/Fm values were high and comparable. The fluidity of the isolated thylakoid membranes at two different depths of the 
membrane was analyzed by EPR with 5-SASL and 16-SASL spin labels. 5-SASL allows to monitor the fluidity in the lipid head groups area and due to 16-SASL, insight into the hydrophobic region of the membrane is possible. The fluidity of the head group regions of the thylakoid membranes was stable only in a narrow but physiological range of the tested temperatures $\left(12\right.$ and $\left.20^{\circ} \mathrm{C}\right)$. It indicates a good adaptation capacity of $T$. pseudonana thylakoid membranes to these temperatures. However, in this range of temperatures the polar region of thylakoid membranes isolated from diatoms grown at the higher cultivation temperature showed a clearly higher rigidification compared to membranes isolated from cells adapted to lower temperatures (Fig. 5A).

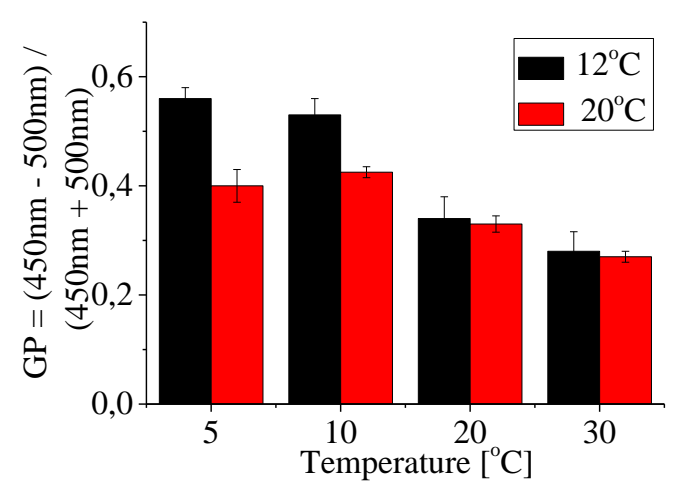

Fig. 6: Effect of growth temperature on GP values of laurdan-labelled thylakoid membranes of T. pseudonana for the temperature range from $5^{\circ} \mathrm{C}$ to $30^{\circ} \mathrm{C}$. The mean $\mathrm{GP}$ values of seven independent thylakoid preparations are presented.

It is worth noticing that membrane fluidity between cells cultured at 12 and $20^{\circ} \mathrm{C}$, in the physiological range of temperatures is strongly different above a temperature of $12^{\circ} \mathrm{C}$. It indicates that $T$. pseudonana has to use its adaptive mechanisms allowing them to obtain the optimal fluidity of the polar membrane region only when diatoms are growing under higher temperatures (Fig. 5A). The fluidity of the hydrophobic region of $T$. pseudonana thylakoid membranes was both stable and comparable in the temperatures range between 12.5 and $20^{\circ} \mathrm{C}$ for both the cultures grown at low or higher temperatures (Fig. 5B). Outside the physiological temperature range, the membrane fluidity of diatoms cultivated at higher temperatures was slightly higher at low measuring temperatures compared to that observed for diatoms acclimated to low temperatures. However, it was clearly lower at higher temperatures used for the EPR measurements. These results were in general confirmed by the laurdan fluorescence method (Fig. 6).

To obtain more direct information about the molecular mechanism of photosynthetic membranes adaptation to changing temperatures, the fatty acid composition was analyzed (Fig. 7).

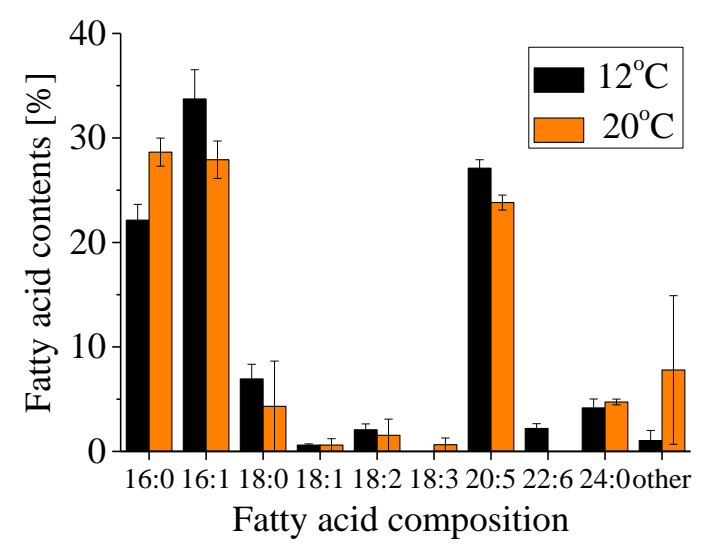

Fig. 7: Effect of cultivation temperature on fatty acid composition of T. pseudonana grown at different temperature $\left(12\right.$ and $\left.20^{\circ} \mathrm{C}\right)$ under the same light conditions $\left(40 \mu \mathrm{mol} \mathrm{m}^{-1} \mathrm{~s}^{-1}\right)$ in batch cultures. Values are the average of three experiments ( \pm standard error). 
The most dominant fatty acids of T. pseudonana were palmitic (16:0), palmitoleic (16:1), and the polyunsaturated fatty acids: eicosapentaenoic (20:5n3) and decosahexanoic (22:6n3) acid (Fig. 7) which are not found in vascular plants. The decrease of the $T$. pseudonana growth temperature from moderate to $12^{\circ} \mathrm{C}$ caused a decrease of the saturated $(16: 0$ and 24:0) and an increase of the unsaturated fatty acids. Such changes play an essential role in the maintenance of the fluidity of the hydrophobic membrane regions $[15,36,37]$ thereby providing stability to the metabolomic processes which take place within the membrane, such as photosynthetic light-harvesting and electron transport [38]. Additionally, we observed an increase of the EPA contents in T. pseudonana cells when cultures were transferred from 20 to $12^{\circ} \mathrm{C}$. During adaptation, the level of EPA in the T. pseudonana cells increased by about $14 \%$. Additionally, the presence of decosahexenoic acid was detected.

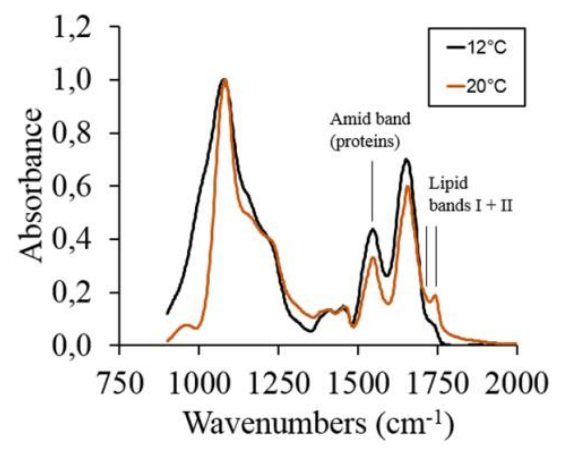

Fig. 8: Effect of culture growth temperature $\left(12\right.$ and $\left.20^{\circ} \mathrm{C}\right)$ on the protein to lipid ratio, analyzed by FT-IR spectroscopy in thylakoid membranes of $T$. pseudonana on the eighth day of culture, under the same light conditions $\left(40 \mu \mathrm{mol} \mathrm{m}^{-1} \mathrm{~s}^{-1}\right)$.

Changes in unsaturation degree of fatty acids are not the only mechanism which affects the membrane fluidity. Environmental temperature changes were shown to also induce modulation of the membrane composition by regulation of the protein concentration and the lipid:protein ratio [29]. The FT-IR analysis of the thylakoid membranes showed an increased lipid:protein ratio in thylakoid membranes of $T$. pseudonana cells cultured at higher temperature (Fig. 8), what also affected the membrane fluidity [39]. Additionally, we also observed an increase of the protein:chlorophyll as well as the protein: $\mathrm{OD}_{600}$ ratio in intact $T$. pseudonana cells cultured at lower temperatures (Fig. 3).

\section{Conclusion}

The obtained results present the adaptation strategies of the model diatom species, T. pseudonana to the changes of the important environmental factor temperature. The data shows that regulation of the content and interaction of biomolecules such as fatty acids, proteins and pigments is the most important molecular strategy in the optimization of the diatom photosynthetic membranes under changing environmental temperatures. Obtained results show also the significance of the membrane fluidity regulation mechanisms for the diatom adaptation to the greenhouse effect. They also indicate that the mechanisms for the regulation of membrane fluidity are different for the polar regions and the hydrophobic area of photosynthetic membranes.

\section{Acknowledgements}

This work was supported by project No. 2011/01/M/NZ1/01170, KNOW - The Faculty of Biochemistry, Biophysics and Biotechnology of Jagiellonian University a partner of Leading National Research Center (KNOW) supported by the Minister of Science and Higher Education No. A1/183 and by a grant from the DFG (Go 818/7-2).

\section{References}

[1] S. Levitus, J. I. Antonov, T. P. Buyer, C. Stephens, "Warming of the World Ocean," Science, vol. 287, no. 5461, pp. 2225-2230, 2000.

[2] J. R. Toggweiler, J. Russel, “Ocean circulation in warming climate," Nature, vol. 451, no. 17, pp. 286-288, 2008.

[3] E. V. Armbrust, "The life of diatoms in the world's oceane," Nature, vol. 459, no. 14, pp. 185-192, 2009. 
[4] P. G. Falkowski, R. T., Barber V. Smetacek, "Biochemical controls and feedbecks on ocean primary production," Science, vol. 281, pp. 200-206, 1998.

[5] A. M. Pyszniak, S. P. Gibbs, "Immunocytochemical localization of photosystem I and the fucoxanthin-chlorophyll a/c light-harvesting complex in the diatom Pheodactylum tricornutum," Protoplasma, vol. 166, pp. 208- 2017, 1992.

[6] C. Wilhelm, C. Büchel, J. Fisahn, R. Goss, T. Jacob, J. LaRoche et al., "The regulation of carbon and nutrient assimilation in diatoms is significantly different from green algae," Protist, vol. 157, pp. 91-124, 2006.

[7] B. Lepetit, R. Goss, T. Jacob, Ch. Wilhelm, "Molecular dynamics of the diatom thylakoid membrane under different light condition," Photosynth. Res., vol. 111, pp. 245-257, 2012.

[8] B. Lepetit, D. Volke M. Gilbert, Ch. Wilhelm, R. Goss, "Evidence for the existence of one antenna-associated, lipiddissolved and two protein-bound pools of diadinoxanthin cycle pigments in diatoms," Plant Physiol., vol. 154, pp. 1905-1920, 2010.

[9] R. Goss, T. Jakob, "Regulation and function of xanthophyll cycle-dependent photoprotection in algae,"Photosynth Res. vol. 106, pp. 103-122, 2010.

[10] R. Goss, B. Lepetit, "Biodiversity of NPQ," J. of plant Physiol., vol. 172, pp. 13-32, 2015.

[11] L. Premvardhan, B. Robert, A. Beer, C. Büchel, "Pigment organization in fucoxanthin chlorophyll a/c(2) proteins (FCP) based on resonance Raman spectroscopy and sequence analysis," Biochim. Biophys. Acta, vol. 1797, pp. 1647-1656, 2010.

[12] R. Goss, J. Nerlich, B Lepetit, S. Schaller, A. Vieler, Ch. Wilhelm, "The lipid dependence of diadinoxanthin deepoxidation presents new evidence for a macrodomain organization of the diatom thylakoid membrane," J. of Plant Physiol., vol. 166, pp. 1839-1854, 2009.

[13] H. Abida, L. J. Dolch, C. Meï, V. Villanova, M. Conte, M. A. Block, G. Finazzi, O. Bastein, L. Tirichine et al., "Membrane glycerolipid remodeling triggered by nitrogen and phosphorus starvation in Phaeodactylum tricornutum," Plant Physiol., vol. 167, pp. 118-136, 2015.

[14] M. L. Hamilton, J. Warwick, A. Terry, M. J. Allen, J. A. Napier, O. Sayanova, "Towards the industrial production of omega-3 long chain polyunsaturated fatty acids from a genetically modified diatom Phaeodactylum tricornutum," PLOS one, vol. 10, no. 12, e0144054, 2015.

[15] H. I. Li, Y. L. Lu, J. W. Zheng, W. D. Yang J. S. Liu, "Biochemical and genetic engineering of diatoms for polyunsaturated fatty acid biosynthesis," Mar. Drag., vol. 12, pp. 153-166, 2014.

[16] M. Bojko, M. Olchawa-Pajor, M. Chyc, D. Latowski, "Effect of temperature on composition and fluidity of Phaeodactylum tricornutum thylakoid membranes," Acta Biochomica Polonica, vol. 63, pp. 17, 2016.

[17] E. V. Ambrust, J. A. Berger, Ch. Bowler, B. R. Green, D. Martinez, N. H. Putman, S. Zhou, A. Allen et al., "The genome of the diatom Thalassiosira pseudonana: ecology, evolution, and metabolism," Science, vol. 306, pp. 79-85, 2004.

[18] R. R. L. Guillard, J. H. Ryther, "Studies of marine planktonic diatoms. I. Cyclotella nana Hustedt and Detonula confervacea Cleve," Can. J. Microbiol., vol. 8, pp. 229-239, 1962.

[19] M. Bojko, K. Brzostowska, P. Kuczyńska, D. Latowski, M. Olchawa-Pajor, W. Krzeszowiec, A. Waloszek, K. Strzałka, "Temperature effect on growth, and selected parameters of Phaeodactylum tricornutum in batch cultures," Acta Biochimica Polonica, vol. 60, no. 4, pp. 861-864, 2013.

[20] W. Yongmanitchai, P. Ward, "Growth of Omega-3 fatty acid production by Phaeodactylum tricornutum under different culture conditions," App.l Environ. Micriobiol., vol. 57, pp. 419-425,1991.

[21] O. H. Lowry, N. J. Rosebrough, A. L. Farr, R. J. Randall, "Protein measurement with the Folin phenol reagent," J. Biol. Chem., vol. 193, pp. 265-275, 1951.

[22] S. W. Jeffrey, G. F. Humphre, "New spectrophotometric equation for determining chlorophyll a, b, c1 and c2," Biochem. Physiol. Pflanz., vol. 167, pp. 194-204, 1975.

[23] B. Lepetit, D. Volke, M. Mila'n Szabo, R. Hoffmann, G. Garab, Ch. Wilhelm, R. Goss, "Spectroscopic and molecular characterization of the oligomeric antenna of the diatom Phaeodactylum tricornutum," Biochemistry, vol. 46, pp. 9813-9822, 2007.

[24] K. Strzałka, I. Hara-Nishimura, M. Nishimura, "Changes in physical properties of vacuolar membrane during transformation of protein bodies into vacuoles in germinating pumpkin seeds," Biochim. Biophys. Acta, vol. 1239, pp. 103-110, 1995. 
[25] P. Stępien, A. Pilot, A. Wisniewska-Baker, "Comparative EPR studies on bilayer properties in nanodiscs and liposomes," Biochim. Biophys. Acta, vol. 1848, pp. 60-66, 2015.

[26] D. Marsh, "Electron Spin Resonance: Spin Labels", in Membrane Spectroscopy, E. Grell, Ed., Springer Verlag, Berlin, pp. 51-142, 1981.

[27] K. Ichihara, Y. Fukubayashi, "Preparation of fatty acid methyl esters for gas-liquid Chromatography," Journal of Lipid Research, vol. 51, pp. 635-640, 2010.

[28] T. Parasassi, G. De Stasio, A. d'Ugaldo, E. Gratton, "Phase fluctuation in phospholipid membranes revealed by Laurdan fluorescence," Biophys. J., vol. 57, pp. 1179-1186, 1990.

[29] D.A. Los, K.S. Mironov, "Regulatory role of membrane fluidity in gene expression and physiological functions," Photosynth. Res., vol. 116, pp. 489-509, 2013.

[30] I. Kudo, M. Miyamoto, Y. Maita, "Combined effects of temperature and iron on the growth and physiology of the marine diatom Phaeodactylum tricornutum (Bacillariophyceae)," J. Phycol., vol. 36, pp. 1096-1102, 2000.

[31] D. S. Nichols, J. Olley, H. Garda, R. R Branner, T. A. McMeekin, "Effect of temperature and salinity stress on growth and lipoid composition of Shewanella gelidimarina," Appl. Environ. Microbiol., vol. 66, pp. 2422-2429, 2000.

[32] N. Domingues, A. R. Matos, J. M. de Silva, P. Cartaxana, "Response of diatom Pheodactylum tricornutum to photooxidative stress resulting from high light exposure," PLOS one, vol. 7, no. 6, e38162, 2012.

[33] A. K. Matto, H. Hoffamn-Falk, J. B. Marder, M. Edelman, "Regulation of protein metabolism: coupling of photosynthetic electron transport to in vivo degradation of the rapidly metabolized 32-kilodalton protein of chloroplast membrane," Proc. Nat Acad Sci USA, vol. 81, 1380-1384, 1984.

[34] Z. Gombos, E. Kenero, N. Tsvetkova, T. Sakamoto, E. M. Aro, N. Murata, "Genetic enhancement of the ability to tolerate photoinhibition by induction of unsaturated bonds into membrane glycerolipids," Plant Physiol., vol. 115, pp. 551-559, 1997.

[35] N. Murata, S. Takahashi, Y. Nishiyama, S. I. Allakhverdiev, "Photoinhibition of photosystem II under environmental stress," Biochim. Biophys. Acta, vol. 1767, pp. 414-421, 2007.

[36] J. L. Harwood, "Fatty acid metabolism," Ann. Rev. Plant Physiol. Plant Mol. Biol, vol. 39, pp. 101-138, 1988.

[37] S. Renaud, H. Zhou, D. Parry, L. V. Thinh, K. Woo, "Effect of temperature on the growth, total lipid content and fatty acid composition of recently isolated tropical microalgae Isochrysis sp., Nitzschia closterium, Nitzschia paleacea and commercial species Isochrysis sp.," J. Appl. Phycol., vol. 7, pp. 595-602, 1995.

[38] Z. Gombos, H. Wada, E. Hideg, N. Murata, "The Unsaturation of Membrane Lipids Stabilizes Photosynthesis against Heat Stress," Plant Physiol., vol. 104, pp. 563-567, 1994.

[39] I. Jajić, A. Wisniewska-Bacer, T. Sarna, M. Jemioła-Rzemińska, K. Strzałka, "EPR spin labeling measurement of thylakoid membrane fluidity during barley leaf senescence,” J. of Plant Physiol., vol. 171, pp. 1046-1053, 2014. 